RESUMO Na capa da edição de junho de 2007, a revista Nature celebrava os 50 anos da interpretação dos muitos-mundos da mecânica quântica. Essa capa serviu para ilustrar o prestígio atual que essa interpretação possui, sendo estudada por filósofos e físicos. Todavia a história do seu desenvolvimento e de sua origem permaneciam problemas em aberto na historiografia. Nesse trabalho, nos dedicamos a examinar as origens históricas dessa interpretação durante o doutorado de Hugh Everett, entre 1953-1957. O desenvolvimento dessa interpretação levou a um debate entre John A. Wheeler, orientador de Everett, e Niels Bohr, criador da interpretação da complementaridade, tendo como resultado o encerramento da carreira de Everett na Física. O conflito com Bohr e o modo como esse debate foi conduzido ajudaram truncar a carreira de Everett na física e relegar a sua interpretação ao esquecimento, ao menos durante os 15 anos seguintes.

Palavras-chave: teoria quântica; controvérsias científicas; história da Física; Hugh Everett; John A. Wheeler; Niels Bohr.

\begin{abstract}
Nature celebrated, on the cover of its 2007 June issue, the 50th anniversary of the many-worlds interpretation of quantum mechanics. The cover illustrated the current status of this interpretation, widely studied by physicists and philosophers. However, the history of its development and origins remained an open problem in the historiography of physics. This paper is dedicated to exam the historical origins of this interpretation during Hugh Everett's doctoral work at Princeton between 1953-57. This work led John A. Wheeler, and the creator of the complementarity interpretation, Niels Bohr, into a debate. Bohr's opposition to Everett's ideas, and the way the debate was conducted, contributed to the end of Everett's career in Physics and left his interpretation forgotten at least for the next 15 years.
\end{abstract}

Key words: quantum theory; scientific controversies; history of physics; Hugh Everett; John A. Wheeler; Niels Bohr.

\section{Para que serve uma função} de onda?: Everett, Wheeler, Bohr e uma nova interpretação da teoria quântica*

What is a wave function good for?: Everett, Wheeler, Bohr and a new interpretation of quantum theory
OLIVAL FREIRE JR.

Universidade Federal da Bahia | UFBa

\title{
Apresentação
}

A interpretação dos estados relativos, também conhecida como interpretação dos "muitos-mundos", publicada em 1957 por Hugh Everett, é hoje uma das principais linhas interpretativas da teoria quântica. Qualquer pesquisador envolvido com o tema não pode estar alheio a ela, mesmo que seja para criticá-la. Sua importância cresceu tanto ao longo dos tempos que recentemente, em julho de 2007, por ocasião dos 50 anos da publicação do artigo original dessa interpretação, a conceituada revista Nature dedicou a capa (ver Figura 1), o editorial e dois artigos para discutir essa interpretação.

Essa capa traz uma alegoria à ficção científica dos anos 1950, sugerindo a interpretação de Everett como uma idéia que originalmente parecia se encaixar como ficção científica mas que hoje é parte da ciência estabelecida. ${ }^{1}$ A situação é tal que eventualmente algum partidário chega a afirmar que hoje essa interpretação é consensual. ${ }^{2}$ Essa informação não parece corresponder à realidade, já que, como Freire Jr. afirma, "aqueles físicos [da década de 70] reconheceram a existência de uma controvérsia científica acerca dos fundamentos e 
interpretações da mecânica quântica", ${ }^{3}$ situação que permanece até hoje, tendo completado mais de oito décadas de discussões. ${ }^{4}$ Mas, sendo ou não um consenso, sua importância na Física contemporânea não diminui. Nem todas as implicações filosóficas e físicas dessa interpretação estão bem esclarecidas, e pesquisadores têm se esforçado tanto para esclarecer o significado e as implicações epistemológicas dessa teoria bem como para buscar possíveis experimentos que possam diferenciá-la das outras interpretações ou mesmo situações experimentais cujos significados seriam compatíveis somente com essa interpretação. ${ }^{5}$

Ainda assim, a sua história permanece um problema em aberto. ${ }^{6}$ Poucos estudos abordaram esse tema e diversos pontos permanecem como lacunas. Podemos, em especial, destacar dois destes: o processo de surgimento dessa interpretação e o conseqüente debate que ela suscitou antes mesmo de sua publicação; e como nas décadas de 1970 e 80 os estudos de descoerência foram fortemente influenciados por questões relacionadas com essa interpretação. Dentre esses pontos, o nosso trabalho se concentra no debate por ela suscitado. ${ }^{7}$

Além de preencher essa lacuna historiográfica, esse tema, ao se enquadrar como uma controvérsia em desenvolvimento, termina por suscitar um interesse mais amplo. 0 professor Renan Freitas argumenta que "[a] visão de que a história da ciência precisa ser entendida através do exame de controvérsias científicas e de como estas controvérsias vieram a ser resolvidas em circunstâncias determinadas tornou-se uma verdadeira máxima heurística para o historiador da ciência", mas que "[c]ontrovérsias não são importantes por si mesmas, mas apenas na medida em que se aprende algo a partir delas. Se isto é verdade, então, no que toca a controvérsias, as perguntas relevantes são: 0 que se pode aprender de uma dada controvérsia, e qual a importância de tal aprendizado?". ${ }^{8}$ Se, então, precisamos responder tais perguntas, é possível argumentar que não só temos compreendido de modo mais adequado quais foram os termos do debate ocorrido durante a década de 50 sobre fundamentos da quântica, o que possui um interesse mais restrito à comunidade de historiadores da física contemporânea, mas também que o estudo dessa controvérsia tem contribuído para a nossa compreensão de como e por que cientistas desen-

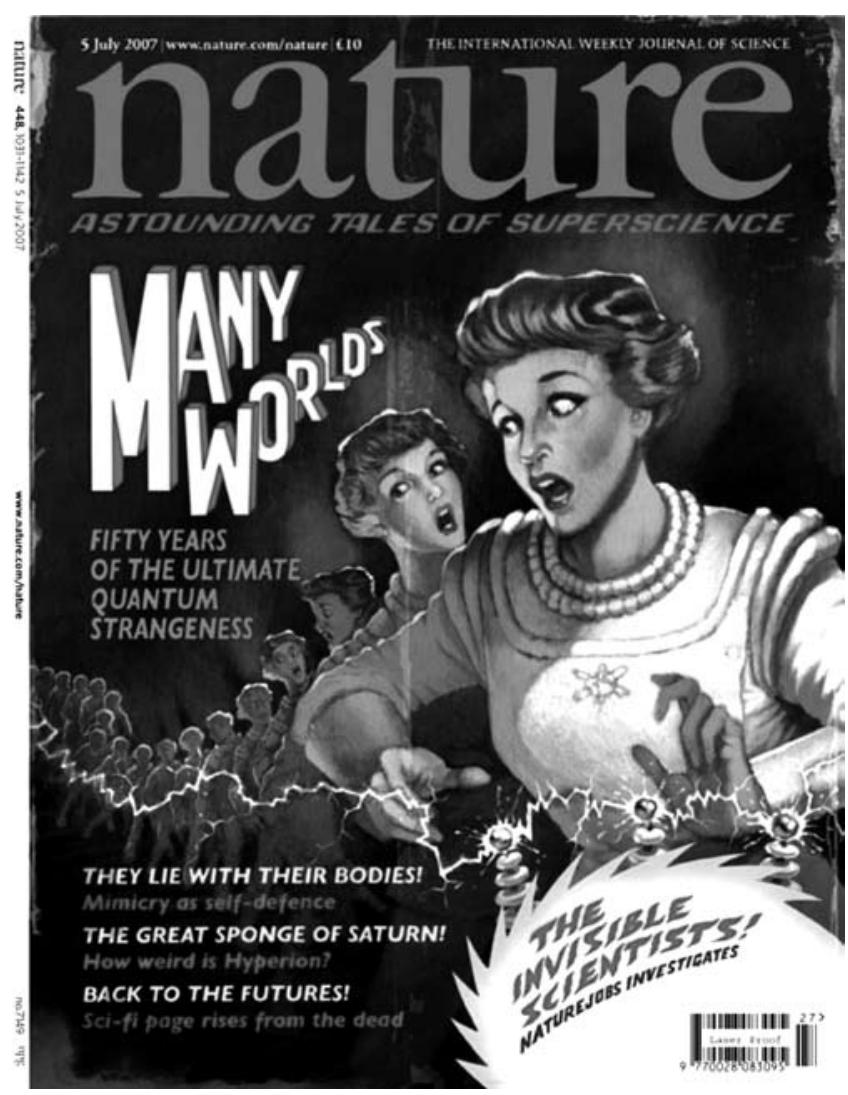

Fig 1 - Capa da Revista Nature de junho/ 2007 por ocasião dos 50 anos da interpretação de Everett. volvem novas teorias, quais os fatores que os influenciam nesse sentido, tanto internos como externos à ciência, e em qual medida eles são condicionantes das formas que as teorias assumem. São esses os elementos que permitem que formemos uma imagem da prática da ciência, mas não de uma ciência bem estabelecida e estática, mas de uma ciência com caráter dinâmico, na qual seus personagens se engajam em debates e utilizam de diversas estratégias para convencer a comunidade e, com isso, "vencer" aquela etapa do debate.

No debate ocorrido em 1956, três foram os principais personagens: Hugh Everett, John Wheeler e Niels Bohr. Hugh Everett, então um estudante de doutorado, orientado por Wheeler, será apresentado posteriormente. John Archibald Wheeler, um dos mais importantes físicos do século XX, é relativamente desconhecido fora do círculo de 
físicos. Durante a década de 30, ele desenvolveu um modelo para o núcleo atômico, em colaboração com Niels Bohr, que seria fundamental no desenvolvimento posterior da bomba atômica por fissão nuclear, durante a segunda guerra. Nos esforços de construção dessa bomba atômica, Wheeler teve um papel importante, mas não tanto quanto o que ele teria na construção da próxima geração, as bombas de fusão nuclear, ou bomba H. Logo no pós-guerra, ele se engajou no desenvolvimento da bomba $\mathrm{H}$ e na tentativa de convencer tanto 0 seu departamento, em Princeton, como o governo americano da necessidade de sair na frente numa possível corrida armamentista, que efetivamente veio a acontecer. Posteriormente, ele deixaria de trabalhar em física nuclear e migraria para a relatividade generalizada, tendo efetuado importantes avanços teóricos, tais como as idéias de Espuma Quântica, Buracos de Minhoca e "Geons". Ele também foi o responsável por cunhar o nome Buraco Negro e um dos pioneiros na busca de uma quantização da gravitação. Na década de 1970 e nos anos posteriores ele voltaria seus interesses de pesquisa para os fundamentos da teoria quântica, sugerindo um experimento de pensamento chamado de experimento de escolha demorada (delayed choice experiment) e sugerindo que o ente mais básico da Física fosse a informação, cunhando o termo "it from bit". Além das suas importantes contribuições teóricas, ele desenvolveu um papel de aglutinar grupos de pesquisa e de incentivar jovens pesquisadores, tendo deixado uma marca importante no modo com que a Física americana se desenvolveu no século XX. Niels Bohr, por outro lado, dispensa grandes introduções. Um dos chamados pais fundadores da teoria quântica, em 1913 ele desenvolveu um novo modelo atômico, postulando a estabilidade das órbitas dos elétrons, tendo sido agraciado com o prêmio Nobel de 1922. Uma de suas contribuições para a Física foi o desenvolvimento da interpretação da complementaridade, uma interpretação que permitia compreender o significado da nova teoria recém desenvolvida em 1926, interpretação esta que, como veremos, está no cerne da controvérsia em questão. Além de importantes contribuições à física teórica, ele exerceu um papel de liderança na Física, seja através de seu carisma ou de sua autoridade, até seu falecimento em $1962 .{ }^{9}$ Além desses, alguns outros personagens participaram do debate. Aage Petersen e Alexander Stern participaram de leituras e seminários e trocaram cartas com Everett e Wheeler sobre a tese. Hip Groenewold teve a oportunidade de ler a versão preliminar da tese e a versão publicada, comentando a transição entre estas, e Léon Rosenfeld, que só dialogaria com Everett posteriormente, em 1959, teve a oportunidade de ler os textos e discuti-los com Bohr.

Nesse debate entre a interpretação de Everett e a de Bohr, Wheeler buscou, por um lado, mostrar para Bohr que essa nova abordagem era uma generalização da sua interpretação, e, por outro, convencer Everett que existiam pontos em sua abordagem que eram incompatíveis com a visão bohriana e que deveriam ser modificados. Como resultado, 0 pensamento de Bohr não foi modificado, mas a tese de Everett sofreu mudanças significativas. 0 exame dos termos nos quais esse debate foi travado servem para compreender um pouco melhor como o pensamento de Bohr era compreendido na década de 1950 e, assim, entender porque Wheeler pensou que era possível compatibilizar ambas as abordagens. Ademais, compreender o papel que John Wheeler, tanto orientador de Everett como amigo de Bohr e 0 principal responsável pelo debate, teve em toda essa história é ilustrativo das relações pessoais dentro da ciência.

Desse modo, neste trabalho nos concentraremos em apresentar a história do surgimento da interpretação dos estados relativos e seu significado e do posterior debate em Copenhague. Primeiro, nós apresentaremos qual foi 0 projeto desenvolvido por Everett e qual a estrutura básica de sua interpretação. Continuaremos examinando as origens da interpretação descrevendo um pouco a formação inicial de Everett e o contexto no qual ele iniciou seu doutorado. Seguiremos, então, abordando a discussão da versão preliminar de sua tese entre Wheeler e os pesquisadores de Copenhague e como isso ocasionou mudanças significativas na versão final da tese de Everett. Para compreender os termos do debate, vamos explicitar os principais pontos de discordância, evidenciando o debate entre realismo everettiano e pragmatismo bohriano e, para desenvolver o nosso argumento, contrastaremos com as críticas recebidas pela interpretação quando o artigo foi enviado para publicação. Com essa comparação poderá se evidenciar que o debate não se travou efetivamente, visto que cada grupo falava um "idioma" distinto. 


\section{A interpretação de Everett ${ }^{10}$}

A insatisfação de Everett com a formulação usual da teoria quântica estava principalmente relacionada com 0 postulado de projeção, um artifício matemático desenvolvido na década de 30 por von Neumann para tentar dar um sentido ao colapso da função de onda no formalismo matemático da teoria quântica. Na concepção descritivista, porém pragmática, que Everett sustentava das teorias físicas, ${ }^{11}$ a tentativa de descrever um sistema quântico que contém um observador faz surgir um paradoxo que posteriormente viria a ser conhecido como paradoxo do amigo de Wigner. ${ }^{12}$ Além disso, como o postulado de projeção supõe um observador externo para que o colapso da função de onda aconteça, não seria possível descrever a própria estrutura do espaço-tempo, ${ }^{13}$ pois "[n]ão há nada fora dele para produzir transições de um estado para outro". ${ }^{14}$ Desse modo, no sentido de resolver esses problemas, ele propõe que eliminemos o postulado de projeção.

Para Everett, a evolução do estado físico de um sistema é sempre linear e determinística, de acordo com a equação de Schrödinger. 0 estado físico do sistema é completamente descrito pela função de onda, e essa "função de onda é tomada como a entidade física básica sem uma interpretação a priori". ${ }^{15}$ Para eliminar o postulado de projeção, Everett termina por também destituir a teoria quântica de sua usual interpretação, associada a Niels Bohr. Sem contar com 0 aporte interpretativo bohriano da teoria, Everett propõe reproduzir as mesmas conseqüências experimentais utilizando somente 0 "formalismo bruto", sem o postulado de projeção introduzido por von Neumann, mas sem os princípios interpretativos sugeridos por Bohr, mostrando que sua interpretação é coerente com a prática dos físicos. Dos resultados obtidos, ele pretende extrair uma interpretação "natural" da teoria. Nesse sentido ele descreve observadores dentro da teoria, utilizando uma versão forte do paralelismo psicofísico, ${ }^{16}$ desenvolve o formalismo às últimas conseqüências, e só então tenta entender o significado do que está sendo descrito e, com isso, faz a conexão com a linguagem comum da experiência. Ao fazer isso, ele acredita que o formalismo está exprimindo a sua própria interpretação. ${ }^{17}$

Para interpretar a conexão do formalismo com a nossa experiência, ele cria a noção de estados relativos. Quando uma interação de medição ocorre entre um observador e um sistema em um nível objetivo, ambos os sistemas passam a estar correlacionados ${ }^{18}$ e 0 observador percebe (mede) o sistema em todos os possíveis auto-estados. Em um nível subjetivo, em cada um dos termos da superposição final, o observador terá medido um resultado específico relativo aos outros resultados do sistema. Com essa noção, nunca existe, de um modo geral, um resultado específico após uma medição. Ao contrário, sempre existirá uma série de resultados, uns relativos aos outros ${ }^{19} \mathrm{e}$ todos igualmente reais. Ele pretende que essa noção seja suficiente para explicar qual o sentido de uma função de onda na qual todos os elementos da superposição permanecem acontecendo objetivamente, mesmo após interações entre sistemas quânticos, e qual a conexão desse resultado com a nossa prática. ${ }^{20}$

\section{A história da Tese ${ }^{21}$}

Hugh Everett III graduou-se em Engenharia Química, em 1953, na Catholic University of America, em Washington, recebendo a distinção magna cum laude. No seu histórico escolar, além das disciplinas de Engenharia, existem algumas de Matemática mais avançada, uma de Física moderna e uma de Filosofia da Ciência. ${ }^{22}$

Em sua carta de recomendação para ingresso no doutorado em Princeton, o professor William Boone, professor de Everett na Catholic University, refere-se a Everett como possuindo um raro talento matemático. Ele afirma que talvez nunca encontre outro estudante ao qual ele possa dar apoio tão completo e irrestrito. Ele adiciona ainda que, apesar de Everett ter feito Engenharia Química, ele cursou diversas disciplinas de Matemática avançada, tendo obtido créditos 
suficientes para tirar um major em Matemática. Escrito à mão no topo da carta, um professor pergunta para Eugene Wigner se ele deve ser aceito, e Wigner responde que seria bom aceitá-lo. ${ }^{23} \mathrm{Em} 1953$, Everett ingressa no doutorado em Princeton, usufruindo de uma bolsa da National Science Foundation (NSF). ${ }^{24}$

Não sabemos ao certo a origem das idéias de Everett sobre Mecânica Quântica e quando ele começou a se preocupar com esses problemas, porém alguns elementos podem nos indicar qual o caminho por ele percorrido. Sabemos que ele, como mencionado, na graduação, no período letivo entre 1951 e 52, cursou uma disciplina de Física moderna. No doutorado ele cursou Introdução à Mecânica Quântica durante o seu primeiro ano, 1953-54. Não sabemos qual era o livro texto utilizado nesse curso, mas é bastante provável que ele tenha tido contato com o livro de mecânica quântica de David Bohm. ${ }^{25}$ Esse livro, anterior à formulação bohmiana das Variáveis Ocultas, apresenta a Mecânica Quântica com uma grande preocupação filosófica, em especial com um detalhado tratamento do problema da medição e do paradoxo EPR. ${ }^{26}$ Em 1955, Everett teve a oportunidade de discutir com Charles Misner, que era seu colega de pós-graduação, e Aage Petersen, assistente de Bohr para assuntos filosóficos, que estava desenvolvendo sua tese sobre fundamentos da teoria quântica. Everett reconhece que ele ainda não tinha uma reflexão suficientemente desenvolvida sobre os fundamentos dessa teoria, ao menos não como Petersen. ${ }^{27}$ Outro fator importante foi o modo de Wheeler fazer física ${ }^{28}$. Nesse período, Wheeler estava se convertendo à pesquisa em Gravitação e se dedicou a levar ao extremo as equações matemáticas desse campo de pesquisa, chegando aos resultados dos "Geons" e "Buracos de Minhoca". ${ }^{29}$ Misner, ao comentar sobre esse período, afirmou: “Ele pregava a idéia de que você deve somente olhar para as equações e se lá estiverem os fundamentos da Física, é porque você seguiu suas conclusões e deve ouvi-las seriamente. Ele estava fazendo isso naquelas soluções das equações de Einstein, como Buracos de Minhoca e Geons"; e Everett respondeu: "Eu devo admitir que [...] está certo, e pode muito bem ter sido totalmente instrumental no que aconteceu" ${ }^{30}$ De algum modo, a interpretação de Everett seria, em essência, levar às últimas conseqüências o formalismo da Teoria Quântica.

Além desses pontos é importante especificar a situação dos fundamentos da teoria quântica naquele período. Na descrição de Freire, a década de 1950, em especial o ano de 1952, com a publicação da interpretação das variáveis escondidas de David Bohm, marca o início do período intermediário na controvérsia octogenária sobre fundamentos da teoria quântica. Nesse período intermediário, acontece o fim da chamada monocracia de Copenhague ${ }^{31}$ e, aos poucos, começa a se estabelecer um ambiente crítico acerca dos fundamentos e da interpretação da teoria quântica. Dessa forma é bastante provável que esse ambiente crítico, juntamente ao trabalho de Bohm de 1952, tenha exercido grande influência na forma com que Everett identificou os problemas e cunhou sua solução. ${ }^{32}$

\section{A primeira versão}

Um pouco mais tarde nesse mesmo ano, Everett apresentou dois manuscritos para seu orientador, ${ }^{33} \mathrm{John} A$. Wheeler. Para compreendermos a resposta de Wheeler, precisamos falar um pouco da relação entre ele e Bohr. Wheeler, em 1934, passou um ano em Copenhague fazendo estudos de pós-doutoramento, estudando a estrutura nuclear. Em sua biografia, ele se refere a Bohr como um mentor, e o respeito e a admiração vêm desde muito tempo. Wheeler terminou sendo um porta-voz de Bohr nos Estados Unidos e sempre nutriu esse sentimento de amizade e admiração desenvolvidos no período de sua estada em Copenhague. Dessa forma é possível compreender a necessidade que Wheeler sentia de ver a interpretação de seu pupilo aprovada por Bohr. Wheeler, que sempre enxergou um valor muito grande na interpretação de Everett e acreditava que ela realmente poderia ser uma generalização do pensamento de Bohr, fez todo o possível para evitar conflitos, tomando a linha de frente de defesa dessa interpretação em Copenhague e sempre incentivando Everett a adequar o seu pensamento e a sua escrita a uma forma que não fosse (muito) conflituosa com o pensamento de Bohr. 
Na resposta que Wheeler envia para Everett através de uma nota, em setembro de 1955, ele afirma que o primeiro artigo, no qual se discutia como efetuar uma medida quantitativa da correlação entre dois sistemas, "me parece praticamente pronto para publicar - onde você o publicaria?". Em relação ao segundo manuscrito, ele diz que se sente "realmente envergonhado [bashful] de mostrá-lo para Bohr na sua presente forma". Wheeler ainda acrescenta que considera 0 texto de grande valor, mas sente-se assim "devido a partes sujeitas a interpretações místicas equivocadas por parte de muitos leitores não preparados" ${ }^{34}$ Esse segundo manuscrito, intitulado Probability in wave mechanics [Probabilidade em mecânica ondulatória], é uma apresentação da interpretação de Everett sem utilizar o formalismo matemático. Seus argumentos são semelhantes aos da tese, porém apresentados de modo mais claro e com metáforas mais fortes. É possível que esse texto tenha sido escrito a pedido de Wheeler para apresentar essa interpretação a Bohr. Sua estrutura parece derivar do texto que foi publicado em 1973 e, se isso é verdade, ajuda a colocar um período mais específico para a primeira versão da tese.

Essa primeira versão parece ter sido concluída no final de 55, pois Everett afirma que "a coisa toda estava pronta naquele outono e inverno" ${ }^{35}$ É nessa versão, publicada com o título The theory of the universal wave function [A teoria da função universal], que Everett apresenta pela primeira vez suas idéias. Nesse texto pode-se dizer que toda a interpretação já estava pronta. Todas as modificações, como veremos adiante, não afetariam o conteúdo de sua interpretação, mas efetivamente a forma de apresentação deste. ${ }^{36}$ Em um texto de 137 páginas, Everett começa apresentando cinco possíveis soluções para resolver os paradoxos que emergem do postulado de projeção. Depois de sugerir que a melhor solução é aceitar a validade irrestrita da Mecânica Ondulatória, ele desenvolve alguns resultados matemáticos para serem utilizados no cálculo de correlações na teoria quântica e deduz a relação do formalismo com a experiência e os resultados estatísticos da teoria quântica usual. Na conclusão, ele volta a discutir as possíveis soluções para os problemas da teoria quântica, incluindo a proposta de Bohr como uma das possíveis, porém afirma que "[e]ssa interpretação sofre do dualismo de aderir a um conceito de 'realidade' [...] no nível clássico, mas de renunciar ao mesmo no domínio quântico". ${ }^{37}$ É verdade que essa tese não tinha como objetivo criticar diretamente Bohr, tanto que, em 1957, ele responde a Petersen afirmando que "as dificuldades em particular da Mecânica Quântica que são discutidas em meu artigo estão mais relacionadas com a forma mais comum (pelo menos nesse país) da teoria quântica, como expressa por von Neumann, por exemplo, e não tanto com a interpretação de Bohr (de Copenhague)". Mas, pela próxima frase, na qual ele afirma que "[a] interpretação de Bohr, para mim, é ainda mais insatisfatória e em bases bem distintas" ${ }^{38}$, podemos ver que ele não nutria grande simpatia pela interpretação de Bohr, e esse primeiro texto certamente expressa isso.

Como essa tese colocava a interpretação de Bohr como uma de várias possibilidades, e ainda por cima como uma opção inadequada, Wheeler não poderia deixar que Bohr lesse algo assim. Dessa forma o texto foi revisado por ambos. Essa revisão do rascunho levaria a uma segunda versão que seria efetivamente enviada para Copenhague. 0 título dessa versão, que deve ter ficado pronta no início de abril de 1956, era Wave Mechanics without probabilities ${ }^{39}$ [Mecânica ondulatória sem probabilidades].

\section{A banca em Copenhague}

Wave Mechanics without probabilities, referida como a segunda versão encadernada da tese foi, então, enviada para Petersen, em 24 de abril desse mesmo ano. Em carta para Bohr, Wheeler agradece antecipadamente os comentários dele e de Petersen, mas indica que tanto o título como outras idéias precisam de uma análise mais profunda. Assim ele pede que deixe essas questões de lado e diz que está mais preocupado "com a questão mais fundamental, se existe alguma escapatória [whether there is any scape] de um formalismo como o de Everett". ${ }^{40}$ 
Wheeler enviou essa carta de Leiden, na Holanda. Ele passou oito meses na universidade de Leiden, de janeiro a setembro, para assumir a cátedra H. A. Lorentz. ${ }^{41}$ No início de maio, Wheeler foi à Copenhague. Nessa ocasião, discutiu com Bohr e Petersen a tese de Everett. Em carta enviada para Everett em 22 de maio de 1956, Wheeler menciona que os três tiveram três longas e acaloradas discussões sobre o rascunho da tese. Ele afirma que enviará notas comentando os pontos específicos, mas que, como resultado dessas discussões, concluiu-se que "seu lindo formalismo da função de onda, claro, permanece inabalado; porém todos nós sentimos que a verdadeira questão são as palavras que devem ser anexadas às quantidades do formalismo" ${ }^{42}$ Esse ponto destacado indica que a fonte de discordância entre as duas interpretações não estava no modo como Everett resolveu os problemas, incluindo o aparato observacional dentro do formalismo, mas no significado disso e no papel que a teoria deveria ter para Everett, incompatível com o pensamento de Bohr, como veremos na próxima seção em mais detalhes. Assim sendo, Wheeler conclui: "Por um lado, sua tese está pronta; por outro, a parte mais difícil do trabalho está apenas começando"; e complementa no pós-escrito: "Eu acho que é bobagem minha [foolish of me] falar de datas até que toda essa questão de problemas seja esclarecida".

Nessa carta também é possível ver o papel central de Wheeler nessa história. Ele afirma para Everett que desejaria ver a tese publicada na Academia Dinamarquesa de Ciências, pois esse seria o local ideal, já que tal publicação legitimaria a interpretação para o mundo sob os auspícios de Bohr. ${ }^{43}$ Poucos dias depois, Wheeler expressaria essa vontade para Bohr. Em telegrama enviado no dia 26 de maio de 1956, ele escreve: "meu grande desejo tese adequada publicação academia dinamarquesa após revisão" . ${ }^{44}$ Para satisfazer esse seu desejo, era necessário tentar compatibilizar as idéias e que Everett compreendesse por completo e enxergasse o caminho de como escrever adequadamente sua dissertação. No seu ponto de vista, isso só seria possível se Everett se reunisse com Bohr. Assim, Wheeler fala para Everett que Bohr "[f]icaria muito satisfeito com uma visita sua de várias semanas para resolver tudo isso [...]. Vá, por favor!". Já para Bohr, no telegrama mencionado, diz que "Everett agora princeton ligou pedindo conferência com você espera voar quase imediatamente". Para que fosse possível esse encontro, Wheeler contribuiria com recursos do seu fundo de pesquisa. Era essencial, para Wheeler, que esse encontro fosse possível: "Eu acho que suas idéias muito originais receberão uma grande discussão. Eu insisto que os probleminhas sejam eliminados antes da publicação, não após". 45

Após a visita de Wheeler a Copenhague, Alexander Stern, um engenheiro americano conhecido de Wheeler que estava então trabalhando com os físicos de Copenhague, ficou responsável por apresentar um seminário sobre a interpretação de Everett e, posteriormente, de enviar uma carta listando os principais pontos de discordância, o que ele fez em 20 de maio. No dia 26 de maio, Wheeler responde a Stern os questionamentos feitos à tese de Everett. Na resposta, é curioso que ele atribua somente a Everett todos os questionamentos à posição de Bohr sobre o problema da medida, afirmando que ele mesmo nunca tivera dúvidas sobre isto. Em suas palavras: "Eu não, de modo algum, questiono a auto-consistência e exatidão do formalismo quanto mecânico atual [...]. Pelo contrário, eu tenho apoiado vigorosamente e espero apoiar no futuro a presente e inescapável abordagem ao problema da medição. Para ter certeza, Everett pode ter tido dúvidas sobre esse ponto no passado, mas eu não". Era necessário, em períodos de questionamentos à citada monocracia, reafirmar seu comprometimento com a visão dominante. A última coisa que Wheeler gostaria é que fosse considerado mais um dissidente quântico. Porém, em notas para si, comenta que "Stern não parece dar a Everett crédito por suficiente audácia" e conclui que "[o]s comentários de Stern de que Everett não entende o processo de medição são irrelevantes, já que apenas significam que H.E. não concorda com Bohr" ${ }^{46}$

Após essa última troca de cartas, Everett se dedicou a transformar a segunda versão de sua tese, Wave mechanics without probabilities, numa que Wheeler considerasse adequada. Nesse período, Everett se mudou de Princeton para Washington para trabalhar no Pentágono. George Pugh, que trabalhou com Everett, ao contar sobre as conversas que ambos tinham durante os almoços entre 1956-58, lembra que Everett "contou que muitas pessoas que haviam revisado suas idéias tinham achado elas difíceis de serem incorporadas nos seus próprios entendimentos. Ele disse 
que estava tendo alguma dificuldade para desenvolver uma versão de sua tese que seu orientador e seus revisores se sentissem confortáveis. De fato, mesmo seu orientador, John Wheeler, pedia a ele para fazer uma reescrita substancial do material". 47

Wheeler, em sua autobiografia, ao falar sobre esse processo, disse que pôde perceber a profundidade dessa tese, porém "achei o rascunho muito pouco compreensível. Eu sabia que se eu havia tido tantos problemas com ele, os outros membros da faculdade no seu comitê teriam ainda mais problemas. Eles não apenas considerariam incompreensível; eles poderiam achar sem mérito. Então Hugh e eu trabalhamos longas horas à noite no meu escritório para revisar 0 rascunho" ${ }^{48}$

0 físico Bryce DeWitt trouxe mais luzes à essa história, afirmando que "o artigo publicado foi basicamente escrito por Wheeler: ele sentou-se com Everett e disse para ele exatamente o que omitir do trabalho maior" ${ }^{49} 0$ trabalho de Everett claramente não satisfazia Bohr e seus seguidores e Wheeler tomou a decisão de participar ativamente da reformulação do trabalho. Mesmo após o debate ele ainda acreditava que seria possível compatibilizar as duas posições e sentou-se com Everett para tentar encontrar a forma adequada. 0 resultado desse processo de reescrita e adaptação foi a transformação de uma longa tese de doutorado de 137 páginas em outra bem mais curta, com somente 36 páginas, apresentada em abril de 1957, cujo título era bastante neutro, On the foundations of quantum mechanics ${ }^{50}$ [Sobre os fundamentos da teoria quântica]. Essa versão final foi examinada no departamento de Física de Princeton por Wheeler e V. Bargmann, tendo sido aprovada, mencionando uma possível contribuição significativa à nossa compreensão dos fundamentos da teoria quântica. A banca de defesa oral foi composta por Wheeler, Bargmann, H. W. Wyld e R. H. Dicke, e aprovou Everett com conceito very good, destacando a sua habilidade matemática. ${ }^{51}$

Com o texto final pronto, Wheeler decidiu que seria interessante escrever um texto explicando essa interpretação. A sua autoridade como físico poderia contribuir para que ao menos a interpretação tivesse a oportunidade de ser lida e debatida. Nesse texto ele explicava o objetivo de Everett e mostrava quais eram as grandes vantagens dessa interpretação, chamando atenção especial para a possibilidade de se trabalhar com a quantização da própria estrutura do universo. 0 texto ${ }^{52}$ foi enviado para ser publicado juntamente à tese. Eles então enviaram pre-prints de ambos os textos para uma série de físicos, tais como Bohr, Rosenfeld, Wigner, Freeman Dyson e Erwin Schrödinger. Essa mesma lista ainda incluiria Bohm e Fritz Bopp, porém, por algum motivo, os pre-prints não foram enviados para eles..$^{53}$ Alguns meses depois, a tese agora chamada The "relative state" formulation of quantum mechanics [A formulação dos "estados-relativos" da mecânica quântica], junto com o texto de Wheeler, seria publicada no Reviews of Modern Physics, numa edição especial com os Proceedings da Chapel Hill Conference, um evento dedicado à área de gravitação, do qual Everett não participou. Para um texto que, na visão de Wheeler, seria tão promissor e causaria tanto impacto na Física que deveria ser publicado nos Anais da Academia Dinamarquesa, sob os auspícios de Bohr, foi uma escolha muito modesta publicá-lo nesse periódico, em especial porque a edição na qual os textos saíram era a publicação dos proceedings de uma conferência sobre gravitação e cosmologia. Desse modo, os interessados em fundamentos da teoria quântica não esperariam encontrar nenhum artigo importante naquela edição, e isso contribuiu para o pouco impacto causado pelo artigo, que recebeu menos de 20 citações na primeira década de publicação. Da forma com que foi publicado, 0 artigo parecia ter seu interesse restrito aos problemas da Cosmologia, longe do ambicioso projeto inicial de uma reformulação completa da teoria quântica. ${ }^{54}$

\section{Incomensurabilidade entre interpretações}

Podemos, então, nos concentrar nos termos em que foi debatida a interpretação de Everett. No cerne desse 
debate está a concepção distinta que ambos sustentavam em relação ao que deveria ser uma teoria física e, desse modo, ao estatuto da teoria quântica e de seus entes formais.

Para Bohr ${ }^{55}$ o vetor de estado serve para prever resultados de medições efetuadas em um contexto experimental bem especificado. Esse vetor de estado nunca tem relação com possíveis propriedades físicas que o sistema possui ou virá a possuir por ocasião de um ato de medição. De modo bem claro, Bohr afirma que "[t]odo o formalismo deve ser considerado como uma ferramenta para derivar predições [...] os símbolos em si, como já indicado pelo uso de números imaginários, não são suscetíveis de interpretação pictórica". ${ }^{56} \mathrm{~A}$ concepção de Bohr é, desse modo, pragmática e só é possível falar de fenômenos quânticos quando se tem completamente especificado o tipo de aparato experimental que será utilizado para medir o fenômeno e, a rigor, só depois que a medição é realizada. Essa contextualidade do fenômeno quântico é necessária para o uso do formalismo, que na sua ausência perde seu sentido. ${ }^{57}$

Em completa oposição, Everett e Wheeler consideravam o vetor de estado como possuindo um papel descritivo do mundo, em sintonia com a leitura que eles faziam de von Neumann. ${ }^{58}$ Para Everett, "[u]m sistema físico é completamente descrito por uma função de estado $\Psi$ " "59, e ele acredita que "mostramos que a nossa teoria baseada na mecânica ondulatória pura, que toma como a descrição básica dos sistemas físicos a função de estado - que supostamente é uma descrição objetiva [...] - pode ser colocada em correspondência com a experiência" ${ }^{60}$ Quando Everett insere o observador no formalismo, ele o faz acreditando que essa é a única opção possível, "[s]e desejamos aderir a descrições objetivas" ${ }^{61}$ A inserção do observador enquanto tal no formalismo se dá pela exigência da validade universal desse formalismo. Nenhuma parte da teoria quântica estabelece qualquer diferença entre observadores, que podem ser simplesmente aparatos experimentais ou qualquer tipo de sistema que permita gravar um resultado, e sistemas quânticos, e, ao levar as exigências da descrição ao extremo, o observador passa naturalmente a ser também descrito pelo formalismo.

As críticas em relação ao postulado de projeção também evidenciam esse caráter descritivista. Somente na perspectiva de utilizar o formalismo para descrever um processo físico é que esse postulado é necessário (e também só nessa perspectiva é que ele possui problemas). ${ }^{62} \mathrm{~A}$ crítica de Everett ao postulado emerge nessa perspectiva e o seu projeto se desenvolve todo dessa forma. Todos os problemas que ele aborda estão relacionados com a possibilidade de uma descrição completa do que está acontecendo, e este é um ponto fundamental na sua abordagem.

Tendo em vista essa incompatibilidade de visão acerca do estatuto do vetor de estado e da concepção do papel de teorias físicas, podemos compreender porque o debate entre Everett e os apoiadores da interpretação de Copenhague se deu em termos distintos aos do debate com os interlocutores do artigo publicado. Wheeler e Everett tentavam convencer Bohr de que a abordagem deles do formalismo era essencial para descrever observadores dentro do sistema usando o formalismo da Mecânica Quântica. Para isso, Wheeler perguntava: "se existe alguma escapatória de um formalismo como o de Everett quando se quer lidar com uma situação na qual vários observadores estão envolvidos, e se quer incluir os próprios observadores no sistema que está para receber análise matemática" ${ }^{63} \mathrm{~A}$ resposta de Copenhague era a de que essa tentativa de desenvolver o formalismo não compreendia a forma com que Bohr resolvia esses problemas. Stern, por exemplo, teria escrito para Wheeler afirmando que Everett não entendia o processo de medição e que seria um ato de fé postular uma função de onda que descrevesse a evolução de estado do universo ${ }^{64}$ Para um bohriano, não faz sentido introduzir o aparato de medição (enquanto aparato de medição) dentro do modelo de um fenômeno, porque esse modelo global que inclui o sistema estudado S e o instrumento de medição M continuaria pressupondo a definição de um aparato experimental para efetuar medidas sobre 0 sistema conjunto $S+M \cdot{ }^{65} E$ se 0 vetor de estado tem como objetivo permitir prever resultados experimentais, certamente não possui sentido tentar descrever o universo inteiro por ele. E é por isso que Petersen afirma que ele e seus colegas de Copenhague sentem dificuldades em enxergar os problemas que Everett tenta resolver, que para eles não existem. Porém a expressão mais 
clara da posição desse grupo vem de Rosenfeld, em 1959. Rosenfeld afirma que "[t]entar (como Everett faz) incluir 0 arranjo experimental no formalismo teórico é perfeitamente sem esperança, já que isso só pode deslocar, mas nunca remover, o uso essencial de conceitos não analisados que por si só tornam a teoria inteligível e comunicável" ${ }^{66}$

A resposta de Wheeler às críticas de Stern pode ser sintetizada em dois curtos trechos de sua carta para Stern: "a função de estado como usada nesse sentido não tem absolutamente nada a ver com a função de estado como usada na discussão comum do problema da medição", e "a conexão [entre função de onda e resultados experimentais] tem como objetivo suprir a possibilidade de um modelo completo para o nosso mundo". ${ }^{67}$

0 argumento usado por Wheeler para dizer que a função de onda "de Everett" não tem nada a ver com a de Bohr expressa a falta de compreensão deste do pensamento de Bohr. ${ }^{68} \mathrm{~A}$ distinção para Wheeler é que, no caso de Bohr, 0 aparato é sempre externo. Já no caso da função de onda do Universo, nunca há aparato externo no sistema. Porém não é que o aparato seja efetivamente externo para Bohr. Essa forma de colocar a condição de Bohr é uma maneira de tentar interpretar o argumento em termos físicos, quando na verdade para Bohr trata-se de um argumento transcendental, ou seja, o papel do aparato é de condição de possibilidade do conhecimento e não pode ser efetivamente expressado em termos físicos, embora seja corrente entre os físicos expressar 0 aparato de observação em termos físicos. ${ }^{69}$ E é nesse ponto que Everett atacava a interpretação da complementaridade, mostrando que ela precisaria ser completada. 0 debate entre os dois grupos ficou centrado na própria questão de qual o sentido da teoria quântica e do significado de seus entes, mas cada lado tinha uma resposta própria incompatível com a do outro grupo. 0 instrumentalismo de Bohr não parecia poder se fundir com o descritivismo de Everett, e o debate permaneceu nesse nível, sem entrar em outros pontos.

Esses outros pontos só emergiriam ao se examinar a teoria de Everett na própria perspectiva descritivista, tentando compreender qual o seu sentido. Em especial as cartas de Norbert Wiener e Bryce DeWitt, em resposta aos pre-prints, começariam esse tipo de análise. Wiener apontaria que o uso da medida de Lebesgue, usada para deduzir as probabilidades, não é possível no espaço de Hilbert. Já DeWitt, entre diversos pontos, não consegue compreender a transição da superposição para o resultado efetivamente medido na teoria de Everett. ${ }^{70}$ Muitas outras questões surgiriam posteriormente, em especial debates sobre como deduzir as probabilidades e sobre como escolher uma base preferencial para escrever a função de onda. 0 desenvolvimento posterior da interpretação de Everett até hoje é muito rico. A perspectiva de utilizar o formalismo da teoria quântica para descrever o mundo parece ser muito mais interessante do que "somente" utilizá-lo para prever resultados experimentais. E essa suposta vantagem parece permanecer mesmo quando nos damos conta de todas as dificuldades que emergem ao tentarmos utilizar essa teoria para descrever o mundo. Mas, seguindo o pensamento de Bohr, tentar utilizar teorias sem se dar conta das condições de possibilidade dessa utilização, ou mesmo tentando deduzir essas pré-condições da própria teoria, faz emergir uma série de paradoxos. De acordo com Osnaghi, essas tentativas ignoram umas das lições mais importantes do pensamento de Bohr: que para obter previsões de resultados de medição não é necessário um formalismo que descreva o sistema quântico. ${ }^{71}$ A teoria de Everett jamais poderia ser uma generalização da complementaridade. Como Rosenfeld afirmou, não havia esperanças para ela, pelo menos no campo do pensamento bohriano.

\section{Epílogo}

0 encontro tão desejado por Wheeler entre Bohr e Everett só aconteceria em 1959, cerca de dois anos depois da publicação da tese na Reviews of Modern Physics. Everett viajou para Copenhague e permaneceu lá por seis semanas para discutir a sua interpretação. Porém parece que o esperado debate não aconteceu. Everett lembra que o único 
resultado dessa viagem foi o seu trabalho com os multiplicadores de Lagrange, utilizados para otimizar cálculos. ${ }^{72}$ Esse resultado matemático, sem implicações para os fundamentos da Física Quântica, foi desenvolvido no hotel e posteriormente serviria para Everett montar sua própria companhia, a Lambda Corp. ${ }^{73}$ Em síntese, da estada em Copenhague nada resultou relativo às questões da mecânica quântica. A lembrança de Rosenfeld ajuda a dar uma dimensão de por que nada resultou do debate: "Falando sobre Everett, nem eu e nem mesmo Niels Bohr poderíamos ter a mínima paciência com ele, quando ele nos visitou em Copenhague há mais de 12 anos atrás para vender as idéias desesperançosamente erradas que ele havia sido encorajado, muito imprudentemente, por Wheeler para desenvolver. Ele era indescritivelmente estúpido e não conseguia entender as coisas mais simples em mecânica quântica" ${ }^{74}$

Após essa viagem, Everett nunca mais trabalharia com temas de teoria quântica, e nem seguiria uma carreira acadêmica. A sua decepção com todo o processo e com a falta de repercussão de sua teoria inovadora o desanimou para a pesquisa em Física. Aliado a isso, estava o ambiente de mobilização da pesquisa para o contexto da Guerra Fria em que se encontravam os Estados Unidos. 0 Pentágono pagava excelentes salários para quem estivesse disposto a investir seu talento em prol da defesa nacional e Everett aceitou o desafio. Ele seguiria nos anos iniciais trabalhando para o Pentágono e, posteriormente, prestando serviços para a Defesa Americana através de suas empresas. ${ }^{75}$

Entre 1957 e 1967, praticamente ninguém se lembrava da existência dessa interpretação, evidenciado pela pequena quantidade de citações recebidas pelo artigo. A abordagem dos estados relativos parecia, assim, fadada ao esquecimento. Mas DeWitt, que havia criticado o pre-print que lhe fora enviado, resolveu investir seus esforços para trazer essa interpretação de volta ao mundo da Física, em um processo cuja análise escapa ao escopo de nosso trabalho. Ele chamou um aluno, Roger Graham, para fazer uma revisão bibliográfica de todos os artigos relacionados com fundamentos da teoria quântica e para apresentar a interpretação de Everett. Em 1967, em um contexto cultural e profissional relacionado aos fundamentos da teoria quântica em plena mudança, ${ }^{76}$ DeWitt escreveu um importante artigo que discutia a quantização da gravidade, afirmando explicitamente que, para interpretar adequadamente os resultados teóricos que ele obtivera, era necessário utilizar a formulação de Everett. Nesse mesmo ano, ele apresentou essa formulação em um congresso, os Battelles Rencontres. Aos poucos, o trabalho de Everett passou a chamar a atenção da comunidade dos físicos e, em 1973, DeWitt publicaria a versão original mais ampla da tese de Everett, que permanecia inédita. ${ }^{77}$ Desde então, essa interpretação, junto com suas variantes, possui um papel cativo nos debates sobre fundamentos da teoria quântica. Sua fortuna parece ter conseguido ultrapassar as dificuldades iniciais e seu legado permanecerá ou na Física ou na História da Física. Somente o tempo encerrará essa questão.

\section{Conclusões}

Nesse processo de elaboração e discussão e avaliação inicial da interpretação dos estados relativos, pudemos identificar três protagonistas. 0 primeiro é Everett, que escreveu sua tese em um período pouco propício para desafios às bases da teoria quântica. Esse período de transição entre a monocracia de Copenhague e a institucionalização da controvérsia sobre fundamentos da teoria quântica não era muito adequado para um jovem físico tentando reformular a teoria quântica. Seu destino acabou sendo desistir da Física. 0 terceiro protagonista foi o grupo dos físicos de Copenhague, que, sob a liderança de Niels Bohr, não era muito simpático a novas interpretações da teoria quântica que pudessem modificar a forma com que se entendia essa teoria. No fim, foram estes os responsáveis pelo julgamento da tese de Everett. A banca em Princeton foi uma formalidade.

0 segundo, e trágico, protagonista foi Wheeler. Nele, identificamos uma espécie de espírito dividido. Por um lado, considerava-se partidário da interpretação de Copenhague. A sua adesão à complementaridade e sua admiração por Bohr 
não permitiam que ele assumisse alguma espécie de conflito com essas idéias. Por outro lado, por não compreender bem o pensamento de Bohr, Wheeler enxergava na proposta de Everett não uma contradição com as idéias bohrianas, mas sua generalização. 0 seu grande desejo era ver as idéias de Bohr e as de Everett num só programa de pesquisa. 0 problema que Wheeler enfrentava era conseguir convencer Niels Bohr e seus colaboradores de que essas idéias eram adequadas e concordantes com seu pensamento e convencer Everett a alterar suas palavras e pontos específicos de suas idéias que aparentemente não concordassem com Bohr. De fato, esse projeto estava tragicamente fadado ao fracasso, porque as idéias são suficientemente incompatíveis, pois um dos pontos fundamentais para a exposição de Everett era considerar a função de onda como uma descrição do estado do sistema, enquanto Bohr considerava esta como um instrumento preditivo para calcular probabilidades de resultados de medições. Além disso, como Bohr não enxergava essa função como descrição do sistema, a maioria dos problemas que Everett se propunha a resolver eram, para Bohr, pseudoproblemas.

Nesta relação conflituosa, perdeu o mais fraco. Bohr não chegou a mudar, mas muitas das idéias que Everett tentou introduzir com sua primeira versão da tese foram completamente modificadas. Parte importante da discussão sobre as interpretações e sobre a visão de Everett acerca do que deveria ser uma teoria física foram excluídas da versão final. Além disso, as próprias explicações do formalismo foram se condensando, de modo que quase nada ficou presente no artigo publicado, sobrando somente parte do formalismo e explicações suficientemente vagas, o que explica em parte a posterior proliferação de interpretações da interpretação de Everett.

\section{Notas e Referências Bibliográficas}

Fábio Freitas é doutorando em Ensino, Filosofia e História das Ciências (UFBa-UEFS) e professor no Departamento de Física da Universidade Estadual de Feira de Santana. Olival Freire Jr. é doutor em História Social e professor do Departamento de Física Geral da Universidade Federal da Bahia. Esta pesquisa recebeu financiamento da CAPES, CNPq, American Institute of Physics e American Philosophical Society.E-mails dos autores: fabiofreitas@gmail.com e freirejr@ufba.br

"Este texto é uma versão modificada do cap 2 da dissertação de mestrado de FREITAS, F. H. A. Os estados relativos de Hugh Everett III: uma análise histórica e conceitual. Dissertação: Mestrado. Programa de Pós-Graduação em Ensino, Filosofia e História das Ciências. Salvador: Universidade Federal da Bahia; Feira de Santana: Universidade Estadual de Feira de Santana, 2007. Versões anteriores desse texto foram apresentadas no Seminário Nacional de História da Ciência, VII Encontro da Associação de Filosofia e História da Ciência do Cone Sul, em seminários internos no programa de Pós-Graduação e no Encontro de Pesquisa do Programa de Pós-Graduação em Filosofia da UFBa. Gostaríamos de agradecer aos gentis comentários de Stefano Osnaghi, Michel Paty, Osvaldo Pessoa Jr., Antonio Augusto Passos Videira, Aurino Ribeiro, Roberto Martins, Ademir Santana e dos colegas da pós-graduação. Adicionalmente agradecemos os comentários e sugestões de parecerista anônimo desta revista.

1 NATURE, Editorial: Parallalel worlds galore. Nature, 448(7149), p. 1, 2007; TEGMARK, M. Many lives in many worlds. Nature, 448(7149), p. 23-24, 2007; BUCHANAN, M. Many worlds: see me here, see me there. Nature, 448(7149), p. 15-17, 2007.

2 Ver, por exemplo, Deutsch no seu best seller A essência da realidade. Na página 250 ele diz que "a história de Everett é realmente a de um jovem pesquisador inovador desafiando o consenso dominante e sendo amplamente ignorado até que [...] sua visão gradualmente torna-se o novo consenso". DEUTSCH, D. A essência da realidade. Tradução: Brasil Ramos Fernandes. São Paulo: Makron Books, 2000.

3 FREIRE JR., 0 . The historical roots of "foundations of quantum physics" as a field of research (1950-1970). Foundations of Physics, 34, 11, p. 1741-1760, 2004.

4 FREIRE JR, 0.; FREITAS, F. Uma controvérsia octogenária. Scientific American Brasil, Coleção Gênios da Ciência, Edição Quânticos: os homens que mudaram a Física, São Paulo: Duetto editorial, 2006.
5 Para uma análise extensiva dos trabalhos sobre a interpretação de Everett, ver BARRETT, J. A. The quantum mechanics of minds and worlds. Oxford: Oxford Univ. Press, 1999. Para experimentos que diferenciariam essa interpretação das demais, ver DEUTSCH, op. cit. Dois trabalhos adicionais foram importantes na compreensão das idéias de Everett: BEN-DOV, Y. Everett's theory and the "many-worlds" interpretation. American Journal of Physics, V. 58, N. 9, 829-832, 1990. e LEHNER, C. A. Quantum mechanics and reality: an interpretation of Everett's theory, Tese, Doutorado em Filosofia, Stanford University, 1997

6 Para mais estudos dedicados às origens da interpretação de Everett, ver OSNAGHI, S.; FREITAS, F.; FREIRE JR, 0 . The birth of the Everettian heresy, apresentado na History of Science Society Annual Meeting, 1-4 Novembro 2007, Washington; e BYRNE, P. The many worlds of Hugh Everett, Scientific American, December 2007, 98-105.

7 Essa lacuna tem sido parcialmente preenchida pelos trabalhos de Freire Jr., nos quais, pela primeira vez na literatura, se menciona o referido debate. Ver FREIRE JR, 2004, e FREIRE JR., O. Science and exile: David Bohm, the Cold 
War and a new interpretation of quantum mechanics. Historical Studies in Physical and Biological Sciences, 36(1), p. 1-34, 2005. 0 segundo tema é 0 atual projeto de pesquisa de doutoramento de um dos autores (Freitas).

8 FREITAS, R. S., Porque estudar controvérsias científicas?, Episteme, v.3, n.8, p.208-221, 1998.

9 Sobre Wheeler, ver WHEELER, J.A. Geons, black holes \& quantum foam: A life in physics, New York: Norton, 1998, e GALISON, P. Image \& logic: A material culture of microphysics. Chicago: The University of Chicago Press, 1997, p. 264-272. Sobre Bohr, ver sua biografia: PAIS, A. Niels Bohr's times in Physics, Philosophy and Polity. Oxford: Oxford University Press, 582 p., 1991

10 Para essa apresentação nos baseamos no artigo publicado em 1957. Para tal, ver EVERETT, $H$. The "relative state" formulation of Quantum Mechanics, Reviews of Modern Physics, 29, 3, Julho de 1957. Para uma apresentação mais completa, ver $01^{\circ}$ capítulo de FREITAS, 2007 e as referências citadas.

11 A concepção descritivista de Everett significa atribuir ao vetor de estado da teoria quântica o papel de descrever o mundo, e essa descrição se dá de modo inde-pendente das condições de observação. Assim, essa é uma interpretação realista. Por outro lado, o realismo de Everett era suavizado tendo em vista que uma teoria científica descreve o mundo somente à medida que ela funcione, porém nunca é possível atribuir realidade (estatuto de verdadeira) a uma teoria física. Nossas teorias são modelos, mas, sendo modelos, devem descrever o mundo. Para o descritivismo de Everett, ver OSNAGHI, S. A dissolução pragmático-transcendental do "problema da medição" em física quântica. Cadernos de História e Filosofia da Ciência, Campinas, Série 3, v. 15, n. 1, p.79-125, 2006. Para a visão de Everett de teorias físicas, ver EVERETT, H. The theory of the universal wave function. In: DeWITT, B., GRAHAM, N. (eds.) The many-worlds interpretation of quantum mechanics. New Jersey: Princeton University Series, 1973, apêndice II.

12 Para esse paradoxo, ver EVERETT, 1973, p. 4-6. A apresentação original dele, inédita, está em Objective vs. subjective probability, Everett Papers, American Institute of Physics. Não é possível afirmar com exatidão a data em que esse texto foi escrito, mas podemos restringi-lo a 1954 ou 55

13 Ou seja, quantizar a gravitação. Esse problema começa a ter relevância na década de 19 50, e Wheeler, foi um dos pioneiros nesse empreendimento.

14 EVERETT, 1957, p. 455.

15 Ibid. Grifos no original.

16 De acordo com von Neumann, deve ser possível descrever o processo de observação, ou qualquer outro evento extra-físico, como se o mesmo acon-tecesse no mundo físico, daí o paralelismo psicofísico. 0 forte é no sentido que ele não lida somente com a observação comumente entendida na Mecânica Quântica, ou medição, mas o próprio ato de observar de um ser consciente, como um ser humano, por exemplo, já que o mesmo é composto, na instância mais básica, de um conjunto de elementos descritos pela $\mathrm{MQ}$, seguindo uma linha reducionista.

17 "Here as always theory itself sets the framework for its interpretation". Ibid.

18 Ou ainda emaranhados, ou, em termos matemáticos, em um estado composto não fatorável.

19 "To any arbitrarily chosen state for one subsystem there will correspond a unique relative state for the remainder of the system". Ibid., grifos no original.

20 Para uma discussão dos problemas com essa interpretação, ver FREITAS 2007, capítulo 1 e suas referências.

21 Uma esboço biográfico interessante, que serve como uma boa introdução à vida de Everett, foi escrita por Eugene Shikhovtsev e está disponível somente na internet: SHIKHOVTSEV, E. Biographical sketch of Hugh Everett III. Disponível em: http://space.mit.edu/home/tegmark/everett/everett.html (acesso em 02/02/2007).
22 Histórico escolar de graduação de Hugh Everett, Alumni File of Hugh Everett III, Seeley G. Mud Manuscript Library, Princeton.

23 Carta de William Boone para H. Taylor, reitor da Pós-Graduação, 17 /4/1953. Alumni File of Hugh Everett III, Seeley G. Mud Manuscript Library, Princeton.

24 Grade Card e Recomendation Letter, Alumni File of Hugh Everett III, Seeley G. Mud Manuscript Library, Princeton.

25 Everett cita esse livro na tese, porém o contato dele com o livro poderia ter sido posterior aos cursos de Mecânica Quântica. Como se vê na nota 31, a influência da obra de Bohm sobre Everett parece ter sido significativa.

26 BOHM, D. Quantum theory. New York: Prentice Hall, 1951.

27 Esse relato foi feito de modo informal por Everett, em uma festa, em 1977, 20 anos após o ocorrido. Conversa de Everett com Misner, 1977, Fita transcrita por E. Shikovtsev, Everett Papers (EP), Box 1, Folder 3. Como se trata de uma narração 20 anos depois do ocorrido, sem cotejamento com outra fonte, é necessário tomá-la com cautela

280 estilo de Wheeler ao fazer física e as possíveis influências sobre seus estudantes ainda não receberam a devida atenção da literatura histórica. Para a autobiografia de Wheeler, ver WHEELER, 1998. Sobre o estilo de Wheeler podemos ainda ver o comentário que DeWitt faz sobre ele, em entrevista para realização da biografia: "Wheeler wanting to see what the theory forces you to do". Entrevista de Bryce DeWitt e Cécille Morette-DeWitt, realizada por Ken Ford, 29/02/1995, Oral History Interviews, Center for History of Physics, American Institute of Physics.

29 BASSALO, J. M. F.; FREIRE JR., 0. Wheeler, Tiomno, e a Física Brasileira. Rev. Bras. Ens. Fis., São Paulo, v. 25, n. 4, 2003.

30 Conversa de Misner com Everett, ver nota 18

31 A monocracia de Copenhague, termo cunhado por Max Jammer, representa o período que vai desde 1927, com o conselho de Solvay "aceitando" a interpre-tação da complementaridade até fins da década de 1940. Esse período, apesar das críticas de Einstein e Schrödinger, foi marcado por uma fortíssima adesão dos físicos à essa interpretação e pelas tentativas de desqualificar as críticas como sendo metafísicas, fora do domínio da Física. Ver FREIRE, 2004, p. 575-6.

32 John Bell apontará semelhanças entre as interpretações de Everett e de Bohm. Em diversos momentos, Everett parece ser bastante simpático a Bohm, quando, por exemplo, ele afirma que "these theories [de variáveis ocultas] are of great theoretical importance" e que "might conceivably become important" (EVERETT, 1973, p. 113). Barrett, em seu livro, afirma que "we shall see that when we try to make sense of the usual interpretations of Everett, we often end up with a theory that is very much like one [interpretação que Bell faz de Everett] or the other [interpretação de Bohm] of these two theories". BARRETT, 1999, p. 148. Para a proximidade, ver 0 cap. 5 do livro de Barrett e os artigos citados de Bell ali mencionados.

33 Junto com esses dois manuscritos, nos arquivos de Everett existe um terceiro. Nele se discute o conceito de probabilidade objetiva como sendo invariante de observador para observador. Com esse critério, ele examina as probabilidades da Mecânica Quântica e, utilizando um raciocínio semelhante ao do paradoxo do amigo de Wigner, conclui que as probabilidades da teoria quântica são subjetivas e discute de modo bastante sucinto três formas de resolver esse problema, sendo uma delas algo próximo das variáveis escondidas. Assim, é possível que esse manuscrito tenha sido algum tipo de trabalho da disciplina de Mecânica Quântica que ele cursou, mas isso é apenas uma especulação, já que o texto não está datado. Objective vs. subjective probability, Everett Papers, Box 1, Folder 6.

34 Os manuscritos são "Quantitative measure of correlation" e "Probability in wave mechanics", Everett Papers, Box 1, Folder 6. A resposta é uma nota manuscrita de Wheeler p/a Everett, 21 de setembro 1955, Everett Papers, Box 1, Folder 5

35 Conversa de Everett com Misner, op. cit.

36 Quase todos os resultados matemáticos contidos nessa versão desapare- 
ceram da versão final, porém eles realmente não eram importantes para 0 significado da interpretação.

37 EVERETT, 1973, p. 111

38 Carta de Hugh Everett para Aage Petersen, 31 de maio de 1957, Everett Papers, Box 1, Folder 8.

39 Essa versão nunca foi mencionada na literatura e até o momento não foi possível determinar se ainda existe alguma cópia e qual a extensão das modificações.

40 Carta de Wheeler (em Leiden, Holanda) para Bohr, 24 de abril de 1956. Wheeler Papers, Series I, Box Boh-Bu, Folder Bohr.

41 Para essa cátedra é convidado anualmente um pesquisador de distinta colabo-ração para a Física desde 1955 até o presente. Outros nomes importantes que assumiram essa cátedra foram Eugene Wigner, Felix Bloch e Léon Rosenfeld.

42 Carta de Wheeler para Everett, 22 de maio de 1956, Wheeler Papers, Series I, Box - Di, Folder Everett.

43 "Since the strongest present opposition to some parts of it comes from Bohr, I feel that the acceptance in the Danish Academy would be the best public proof of having passed the necessary tests." Carta de Wheeler para A. G. Shenstone, 28 de Maio de 1956. Wheeler Papers, Box Di, series \#2.

44 Telegrama de Wheeler para Bohr, Archives for the history of quantum physics, Bohr Scientific Correspondence, 33.

45 Carta de Wheeler para A. G. Shenstone, 28 de Maio de 1956. Wheeler Papers, Box Di, series \#2.

46 Carta de Wheeler para Stern, 25 de maio de 1956, Wheeler Papers, Series V, Relativity Notebook 4, p. 92.

47 Notas de George Pugh sobre Hugh Everett, Everett Papers.

48 WHEELER, 1998, p. 268.

49 DEWITT, B. Resenha de "The fabric of reality" de D. Deutsch. Natural Science [http://naturalscience.com/ns/books/book02.html], 1998. Acessado em $22 / 5 / 04$.

50 Essa versão defendida é idêntica ao artigo publicado em 1957, salvo algumas pequenas mudanças estilísticas. 0 texto original, que não foi submetido à banca, só viria ser publicado em 1973, por iniciativa de Bryce DeWitt, que desde fins da década de 1960 se dedicou ao desenvolvimento dessa interpretação. Ver nota 11 para referência.

51 "Dissertation report" e "Oral Examination Report", Alumni File of Hugh Everett III, Seeley G. Mud Manuscript Library, Princeton.

52 WHEELER, J. A. (1957). Assessment of Everett's "relative state" formulation of quantum mechanics. Reviews of Modern Physics, V. 29, n. 3, 463-465.

53 Mailing list de pre-prints, 10 de março 1957, Everett Papers, Series I, Box 5.

54 Andrés Cassinello, ao esboçar a história da interpretação de Everett, não perce-beu o processo de debate sobre a tese antes da publicação e associou seus objetivos e seu destino, estando sempre ligados ao campo da Cosmologia Quântica. Ele atribui o seu pequeno impacto inicial à falta de desenvolvimentos nesse campo entre 1957-67. Como pudemos notar, os objetivos dessa interpretação eram mais ambiciosos que somente ser uma interpretação útil para a quantização da gravitação, e o seu pequeno impacto posterior se deveu mais ao ambiente intelectual fechado para críticas e à escolha de publicar 0 artigo em um periódico de alcance mais restrito numa edição especial voltada para cosmólogos. Ver CASSINELLO, A. (1994). La interpretación de los muchos universos de la mecánica cuántica: apuntes históricos. Arbor, CXLVIII, N. 584, 47-68.

55 Para compreender o pensamento de Bohr nos apoiamos em OSNAGHI, 2006.

56 BOHR, N. (1948) On the notions of causality and complementarity. Dialectica, 2, 312-319
57 OSNAGHI, 2006, p. 84-5.

58 Não é objetivo desse trabalho analisar a visão de von Neumann da teoria quântica. Para tal, ver o volume de STOLTZNER, M.; RÉDEl, M. (eds.). John von Neumann and the foundations of Quantum Physics. Kluwer, Dordrecht (Vienna Circle Institute Yearbook 8), 2001.

59 EVERETT, 1957, p.141.

60 EVERETT, 1973, p. 109.

61 Ibid., p. 117.

62 "Como tem sido mostrado por diversos autores (cf. por exemplo PARK, 1973; VAN FRASSEN, 1991, cap. 8), se se aceitar uma interpretação puramente preditiva do formalismo quântico, assumindo a natureza intrinsecamente condi-cional das predições da física (ou seja, sua inseparabilidade da 'enumeração das condições externas' que definem univocamente 0 contexto experimental (BOHR, 1939, p. 47)), a redução do vetor de estado não é necessária para as-segurar a objetividade dos resultados das medições". OSNAGHI, 2006, p. 89.

63 Carta de Wheeler para Bohr, op. cit.

64 Carta de Wheeler para Stern, op. cit.

$65 \mathrm{E}$, desse modo, 0 aparato experimental que foi inserido dentro do formalismo não teria o papel de um aparato, mas sim de um sistema quântico qualquer, e passaríamos a chamar de aparato experimental o novo contexto experimental para medir sobre 0 sistema (sistema quântico +0 antigo aparato experimental).

66 Carta de Rosenfeld para Saul Bergmann, 21/12/1959. Rosenfeld Papers, Niels Bohr Archive.

67 Carta de Wheeler para Stern, op. cit.

68 Wheeler não era o único Bohriano que não compreendeu inteiramente Bohr. Heilbron argumenta que a mensagem de Bohr foi sendo diluída no percurso de sua difusão e mesmo colaboradores mais próximos, como Pascual Jordan, nem sempre se posicionaram de acordo com o pensamento bohriano. Sobre o caso dos físicos americanos, Heilbron acrescenta que "Bohr's way of working through personal connections could not carry the citadel of AngloSaxon physics, the United States, where he had few if any close friends and where the prevailing Geist opposed mixing physics and philosophy". Ainda que Wheeler fosse um dos poucos amigos próximos de Bohr nos Estados Unidos, ele ainda assim estava inserido no Geist prevalescente. HEILBRON, J. The earliest missionaries of the Copenhagen spirit. In: GALISON, P.; GORDIN, M.; KAISER, D. (eds.). Science and Society:the history of modern physical science in the Twentieth Century. Vol 4. Quantum Histories. Routledge: New York, 2001.

69 OSNAGHI, 2006.

70 Carta de Norbert Wiener para Everett, 9 de abril de 1957. Carta de Bryce DeWitt para Everett, 7 de maio de 1957. Everett Papers, Series I, Box 5.

71 OSNAGHI, 2006, p. 112.

72 Conversa de Everett com Misner, op. cit.

73 Ibid.

74 "With regard to Everett neither I nor even Niels Bohr could have any patience with him, when he visited us in Copenhagen more than 12 years ago in order to sell the hopelessly wrong ideas he had been encouraged, most unwisely, by Wheeler to develop. He was undescribably stupid and could not understand the simplest things in quantum mechanics."Carta de L. Rosenfeld para F. Balentine, 22/6/1972, Rosenfeld Papers, Niels Bohr Archives. Agradecemos a Anja Jacobseen por ter nos chamado a atenção desta carta e por gentilmente nos enviá-la.

75 Notas de GEORGE PUGH, op. cit.; SHIKHOVTSEV, 2003.

76 FREIRE, 2004.

[ Artigo recebido em 10/2007 | Aceito em 01/2008 ] 